\title{
Über die Behandlung der Uterusgonorrhoe bei Prostituierten.
}

\author{
Von
}

Dr. Franz Parádi,

I. Assistenten.

Die Arbeiten des letzten Jahrzehntes über das Wesen der gonorrhoischen Affektionen, besonders über die Biologie des Gonococcus Neisser, hahen unsere Ansichten über die gonorrhoischen Erkrankungen, besonders über die Bedeutung und Wichtigkeit der Gonorrhoe beim Weibe ganz umgestaltet. Der Nachweis des Gonococeus auch in rom Ausgangspunkte der Gonorrhoe entfernt liegenden Organen erklärt uns viele Krankheiten, deren Entstehung früher unbekannt war, und nur zu bekannt sind die traurigen Folgen, welche die weibliche Gonorrhoe nach sich ziehen kann.

Wenn wir von der Bedeutung und Schwere der Gonorrhoe beim Weibe sprechen, so kommt in erster Reihe die Ute$r$ us gonorrhoe in Betracht, umsomehr, als nach den bisherigen Daten neben der Harnröhre der Uterus am bäufigsten der Sitz einer Gonorrhoe ist und die Schwere der weiblichen Gonorrhoe durch jene Kowplikationen gegeben ist, welche im Anschluß an eine ascendierende Uterusgonorrhoe auftreten und welche, wenn sie auch das Leben direkt nur in seltenen Fällen gefährden, doch den Ausgangspunkt schwerer und unheilbarer Erkrankungen bilden, umsomehr, als sie einer Behandlung gar nicht, oder doch nur in sehr geringem Grade zugänglich sind. 
Auch in prophylaktischer Beziehung ist die Uterusgonorrhoe von der größten Wichtigkeit, da sie lange Jahre bestehen und ihre Ansteckungsfähigkeit behalten kanu, umsomehr, da sie der Therapie viel größere Hindernisse entgegensetzt als die Gonorrhoe der Harnröhre und der Bartholinischen Drïsen.

Es ist daher vollkommen berechtigt, fortwährend nach neuen Behandlungsmethoden zu suchen, statt sich auf jenen bequemen Standpunkt zu stellen, welcher die Uterusgonorrhoe von vornherein für unheilbar erklärt. Es mehren sich die Erfahrungen, daß sich nicht an jede Uterusgonorrhoe eine Erkrankung der Adnexe anschließt, und daß wir bei frühzeitiger Erkenntnis der ersteren und ihrer kausalen Behandlung sehr oft im stande sind, die Gonococcen abzutöten, bevor sie an Orte gelangen, die für unsere Therapie unzugänglich sind, und daß wir auf diese Art nicht nur die Uterusgonorrhoe heilen, sondern auch einer Erkrankung der Adnexe vorbeugen können.

Das Dogma von der Unheilbarkeit der Gonorrhoe beim Weibe, welches ursprünglich von den Gynäkologen ausging, wird in neuerer Zeit auch von einigen Venerologen, so Behrend und $\mathrm{Kromayer}$, betont, welche gerade aus diesem Grunde die Kontrolle der Prostituierten auf Gonorrhoe für überflüssig und nutzlos erklären. Auch wir sind der Meinung, daß für die Prophylaxe der Gonorrhoe die jetzt üblichen Kontrolluntersuchungen keinen Wert haben, doch kommen wir aus ganz anderen Gründen zu diesem Schlusse, wie das die im Vorjahre erschienenen Arbeiten von Prof. Marschalkó $(6-7)$ des weiteren ausführen. Wir sind überzeugt, daß wir die weibliche Gonorrhoe, besonders die der Prostituierten, in einer sehr ansehnlichen Anzahl der Fälle heilen, in einer anderen aber wenigstens in dem Sinne günstig beeinflussen können, daß wir den virulenteren Prozeß zu einem weniger virulenten, folglich weniger gefährlichen umzugestalten vermögen.

Jene Erfahrungen kurz zusammen zu fassen, welche uns zu dieser Überzeugung gebracht haben, ist die Aufgabe der gegenwärtigen Arbeit. 
Um die Frage der Heilbarkeit der weiblichen Gonorrhoe meritorisch zu beantworten, müssen die an dem Krankenmateriale einer größeren Hospitalsabteilung erreichten Erfolge ganz objektiv geprüft werden. Derartige statistische Arbeiten, welche das klinische Material eines größeren Krankenhauses umfassen, sind bisher nur in sehr spärlicher Anzahl veröffentlicht worden, und eben deshalb wagen wir zu hoffen, daß unsere Daten nicht ganz ohne Interesse sein, und vielleicht etwas zur Lösung der strittigen Frage beitragen werden, umsomehr, als es wenige andere klinische Institute gibt, deren Krankenmaterial so geeignet für das Studium der weiblichen Gonorrhoe wäre, als das weibliche Krankenmaterial der Universitätsklinik für Hautund venerischen Krankheiten zu Klausenburg. Abgesehen davon, daß die Prostituiertenabteilung mit 24 Betten im Rahmen unserer Klinik untergebracht ist, besteht daselbst noch eine größere Frauenstation für venerische - größtenteils von der Polizei eingelieferte aber nicht einregistrierte - Frauen. Hierzu kommt noch der Vorteil, daß das venerische Krankenmaterial einer kleineren Stadt (Kolozsvár hat 50.000 Einwohner) weniger so großen Schwankungen ausgesetzt ist, wie dasjenige einer Grolstadt, und besser in Evidenz gehalten werden kann, zumal bei uns die Prostitution fast durchwegs bordelliert ist; so dab die einmal bereits behandelten Patientinnen nicht für immer der Beobachtung entgehen, sondern zumeist öfters ins Krankenhaus gelangen.

Dieser Umstaud spielt bei der Beurteilung der Heilerfolge eine wichtige Rolle.

In Laufe des vergangenen Jalhres beauftragte mich mein Chef, Hr. Prof. v. Marschalkó, das weibliche Gonorrhoematerial der Klinik vom Ende 1897 bis 1900, mit besonderer Riücksicht der Uterinalgonorrhoen zu bearbeiten, und über die bei uns üblichon Methoden der Uterusgonorrhoebehandlung und deren Resultate zu referieren. Linige diesbezügliche Daten hat Hr. Prof. v. Marschalkó bereits (6) veröffentlicht. Seit dem Jahre 1901 wird jede an dit dermatologische Klinik aufgenommene Kranke, ungeachtet dessen, ob sie ad hoc an virulenter Gonorrhoe leide oder nicht, in der gynäkologischen Klinik, bei der Aufnahme und bei der Entlassung, einer gynäkologischen Untersuchung unterworfen, welche Herr Prof. von Szabó persönlich ausführt. Eine Publikation dieser Untersuchungen resp. eine statistische Schichtung der auf dieser Weise auch durch den Gynäkologen kontrollierten Gonorrhoefälle unserer Klinik, wird demnächst erfolgen. da wir bereits über mehrere Hundert solcher Fälle verfuigen. Die Resultate derselben versprechen sehr interessant zu sein, und werden. 
vielleicht etwas zum Ausgleicho der Gegensätze zwischen Dermatologen und Gynäkologen beitragen.

In der gegenwärtigen Arbeit, welche sich ausschließlich mit den Fällen von Uterusgonorrhoen vom Ende 1897 bis 1900 beschäftigt, sind diese Daten noch nicht erhalten. Die statistische Bearbeitung dieser Fälle versprach um so interessanter zu sein, als sie sich zum großen Teil auf die Wirkung eines, bisher noch nicht erprobten antigonorrhoischen Mittels bezieht. Dieses Mittel ist das Natrium lygosinat, welches uns von seinem Erfinder, Hr. Dr. Ru do lf F a bin yi, o. ö. Professor der Chemie an der Universität Kolozsvár, zu Versuchszwecken überlassen wurde, nachdem es im Verlaufe einiger bakteriologischer Versuche eine starke antiseptische. Wirkung gezeigt hatte. ${ }^{1}$ )

Bei der Therapie der Uterusgonorrhoe legen wir das Hauptgewicht auf die lokale Behandlung. Von der rein exspektativen Behandlung, Umschlägen, Eisbeuteln und Abführmitteln etc. erwarten wir nicht alles, wenn wir die damit erreichten Erfolge auch anerkennen. Wir meinen nämlich, daß dies allein nur bei sehr akuten Fällen ausreicht; bei solchen nehmen auch wir von der lokalen Behandlung Abstand. Wenn dagegen die ersten akuten Erscheinungen abgelaufen und keine Temperatursteigerungen mehr vorhanden sind, oder die Kranken von Anfang an mit subakuter Uterusgonorrhoe in das Krankenhaus kommen, so legen wir das Hauptgewicht auf die lokale Behandlung. Selbstverständlich verfahren wir in solchen Fällen mit der nöthigen Vorsicht, und beginnen nicht mit der topischen

1) Das Natr. lygosinat. hat Prof. R. Fabinyi durch Kondensierung des Salizylaldehyds mit Aceton erzeugt. Indem die Kondensierung mit starker Natriumlauge vorgenommen wird, bildet sich das Natriumsalz des Diorthokumarketons (Diorthodioxydibenzalacetons) oder das Natrium lygosinat, welches mit 7 Wassermolekülen in schönen, metallischgrünen, glänzenden Prismen krystallisiert. Es ist in Wasser leicht löslich, die Farbe der Lösung ist rubinrot. $100 \mathrm{~g}$ Wasser lösen bei $18^{\circ} 4^{\circ}$ C. $6.10 g$ bei $37^{\circ}$ C. $10 \cdot 73 g$ (Bestimmungen Prof. Fabinyis). Die Lösung kann, ohne sich zu zersetzen, aufgekocht werden. An kühlem Orte aufbewahrt, zersetzt sie sich auch unter dem Einflusse des Tageslichtes nicht. Säuren zersetzen sie, auch größere Mengen von Kohlensäure. Bei Gegenwart von Säuren bildet sich ein gelber, krystallinischer Niederschlag von Lygosin (Diorthokumarketon) Das Natr. lygosinat bildet mit den Salzen der metallischen Erden, Schwer- und Edelmetalle teils amorphe, teils krystallinische Niederschläge. Es bildet auch mit den Alkaloiden farbige Salze. Nach den Untersuchungen von Dozenten S. Jakabházi, I. Assistenten an dem hiesigen pharmakologischen Institute, ist das Natr. lygosinat ein stark antiseptisches und gährungshemmendes Mittel. In die Zirkulation gebracht, reizt es das Atmungszentrum stark. (Ungarische Zeitschrift für Chemie 1899.) Es wird neuerdings unter dem Namen Lygosin-natrium durch die Vereinigten Chininfabriken Zimmer \& Co. in Frankfurt a/M." in den Handel gebracht. 
Behandlung, solange irgend eine Kontraindikation vorhanden ist, oder setzen wie sie aus, falls solche auftreten. Unsere Behandlungsweise weicht von derjenigen der Gynäkologen, wie sie ron $\mathrm{Fehling}(2)$ näher auseinander gesetzt wurde, einigermaßen ab. Nach Fehling kann man zwar verschiedene adstringierende und antiseptische Mittel zur intrauterinen Behandlung anwenden, doch warnt er vor der Anwendung der Braunschen Spritze. Diese Auffassung begründet Fehling damit, daß die eingespritzte Flüssigkeit, oder der im Uterus befindliche Eiter hiebei durch die Tuben in die Bauchhöhle gelangen, und dort Peritonitis verursachen könnte; ferner könnten tetanusähnliche Kämpfe, Thromben in den Uterusvenen und Embolien mit tödlichem Ausgang entstehen. Auf diese Frage können wir hier nicht näher eingehen, nur so viel sei bemerkt, daß alle diese abschreckenden Möglichkeiten höchstwahr'scheinlich nur dann eintreten können, wenn eine größere Menge Flüssigkeit unter starkem Druck in die Uterushöhle gelangt, und für den Rückfluß derselben nicht gesorgt ist. Bei der neueren Form der Braunschen Spritze, mittels welcher wir eigentlich keine Einspritzung, als vielmehr eine Einträufelung vornehmen, ist es kaum vorzustellen, wie Flüssigkeit durch die Tuben in die Bauchhöhle gelangen soll, da die eingespritzte Flüssigkeit und das Sekret hinter der zurückgezogenen Kanüle ausfließen kann.

Daß wir die Bebandlung jedesmal auch auf das $\mathrm{Cavum}$ u teri ausdehnen, geschicht keinesfalls deshalb, als wären wir der Meinung, daß reine Zervikalgonorrhoen zu den Seltenheiten gehören. Wir glauben aber, daß die schmale Schleimhautfalle, welche das Os internum bildet, nur eine anatomische Grenze zwischen Zervikalkanal und Cavum uteri vorstellt, und dem Durchdringen der Gonokokken ebensowenig hinderlich ist, wie z. B. beim Maune der Kompressor das Weitergreifen des Gonorrhoischen Prozesses auf die Pars posterior uretrae nicht hintanzuhalten vermag. Über sichere Untersuchungsmethoden aber, um beim Erkranktsein des Zervikalkanals die Gonorrhoe der Uterusschleimhaut bestimmt auszuschließen, verfügen wir vorläufig nicht.

Auf die klinischen Erscheinungen können wir uns bei der Entscheidung dieser Frage nicht verlassen. Bei einer sehr akuten und schnell ascendierenden Gonorrboe gestatten zwar die ausgesprocheneren subjektiven Erscheinungen, die Temperaturerhebungen, eventuelle Schüttelfröste etc. eine Uteruserkrankung mit ziemlicher Bestimmtheit anzunehmen; allein kommen solche sehr akuten Fälle viel seltener zur Beobachtung (und hauptsächlich bei unserem Material), als die gleich rom Anfang her subakut, schleichend einsetzenden oder gar die chronischen, keine subjektiven Symptome aufweisenden 
Uterusgonorrhoen. Bei solchen lassen uns aber die klinischen Erscheinungen im Stich.

Nach Schultz (14) kann man in ungefähr $38 \%$ von Cervixgonorrhoefällen auch Unterusgonorrhoe feststellen. Seine Daten hat er durch die mikroskopische Untersuchung von durch Aspiration gewonnenem Sekret erhalten. W ertheim (18) hält die Sekretuntersuchungen zu diesem Zwecke nicht für einwandfrei, da eine Beimischung des Cervikalsekretes zum Uterussekret sich selbst bei der größten Vorsicht nicht sicher vermeiden läßt. Wir sind ja auch der Meinung, daß die Gonokokken eben anläßlich einer solchen explorativen Untersuchung in das Cavum uteri verschleppt werden können. Nur wenn man im Gewebe der (mit der Curette abgekratzten) Uterusschleimhaut selbst Gonokokken findet, könne man nach Werthe im von einer Uterusgonorrhoe sprechen.

Diese Methode ist natürlich nur dann anwendbar, wenn die subjektiven Erscheinungen den operativen Eingriff erlauben; ist der Uterus sehr empfindlich, so muß man davon absehen. Ihre Zuverlässigkeit kann aber auf Grund der Schultzschen Untersuchungen ebenfalls angezweifelt werden, da im Laufe derselben bei mehreren Fällen, wo die Diagnose Uterusgonorrhoe unzweifelhaft war, in der abgekratzten Schleimhaut keine Gonokokken nachweisbar waren.

Es ist also unseres Erachtens richtiger bei der Uteringonorrhoe der Prostituierten, wenigstens hinsichtlich der Therapie keine scharfe Grenzen zwischen Zervikal- und Uterusgonorrhoe zu ziehen, sondern überall, wo im Zervikalsekrete Gonokokken sind, vom Anfang an auch das Cavum uteri zu behandeln.

Wir gehen ja aber bei der Behandlung mit der nötigen Vorsicht um. Vor Beginn derselben überzeugen wir uns durch gynäkologische Untérsuchung, ob der Zustand des Uterus und der Adnexa eine intrauterine Behandlung ermöglicht. Wir gebrauchen hierzu flüssige Mittel, welche mittels B raunschen Spritzen (neuere Form, $2 \mathrm{~cm}^{3}$ Inhalt, aufschraubbare Silberkanüle) in die Uterushöhle deponiert werden.

Nur ausnahmsweise wurde die Behandlung mittels Playfair oder Sänger schen Sonden durchgeführt.

Die intrauterinen Injektionen werden nun in der Weise vorgenommen, daß wir mittels der Braunscher Spritze, deren Kanüle jedesmal unmittelbar vorher durch die Flamme gezogen wird, nach vorherigem sorgfältigem Abspülen der Vagina und Portio, und Abtupfen dieser letzteren mit zuerst in Alkohol, dann in Sublimat getauchten Wattetampons, also bei strenger Asepsis, und nach eventueller vorheriger Erweiterung des Os internum mittels $\mathrm{H}$ eg arschen Sonden, damit die Kanüle leicht 
in das Carum uteri dringen, und die injizierte Flüssigkeit gut zurïckfließen könne, bei Rückenlage und $\mathrm{N}$ e u ge ba u e r scher Spekula vorsichtig in das Cavum uteri vordringen, und mittels ganz leisem Druck, bei gleichzeitigem Zurückziehen der Kanüle $1 \mathrm{~cm}^{3}$ Flüssigkeit in die Uterushöhle deponieren und mit dem restlichen $1 \mathrm{~cm}^{3}$ den Zervikalkanal abspülen.

Einige Tropfen der in der Spritze befindlichen Flüssigkeit, welche wir unmittelbar vor der Einführung durch die erhitzte Kanüle pressen, kühlen dieselbe ab und verhindern, daß Luft mit in die Uterushöhle gelange. Auf eine rorsichtige, und nicht gewaltige Einführung der Kanüle, damit die Schleimhaut des Cervix oder Endometriums nicht verletzt werde, sind wir besonders bedacht. Deshalb muß die Flexionsrichtung sorgfältig beriucksichtigt werden.

Bei stärkerer Flexion des Uterus kann die Knickung dadurch ausgeglichen werden, daß man mit einer Kugelzange das vordere Labium erfabt und vorsichtig abwärts zieht. ${ }^{1}$ ) Im Falle eines Hindernisses sucht man ohne stärkeren Druck und vorsichtig vorwärts zu kommen, indem man die Krümmung der Kanüle der Flexionsrichtung der Cervix anzupassen sucht. Einen eventuellen Krampf des Os internum sucht man vorsichtig zu überwinden.

Bei genügender Übung und guter Einstellung der Portio gelingt die Einführung in der Regel sehr leicht; wir waren in der Tat nur in Ausnahmsfällengezwungen, uns der Heg a r schen Dilatatoren $z u$ bedienen.

Die Einspritzungen werden zweimal wöchentlich vorgenommen. In der letzten Zeit haben wir in einigen Fällen die Injektionen oder eher Instillationen mit Natrium lygosinat auch in kürzeren Intervallen vorzunehmen versucht, und fanden, daß dieselben ebenfalls gut vertragen wurden.

Es sind jetzt Untersuchungen im Zuge, ob solche $\mathrm{Pa}$ tientinnen, bei welchen die intrauterine Instillation von Natriumlygosinat sonst keine Reaktion herrorruft, auch eine, jeden dritten oder gar jeden zweiten Tag vorgenommene kinspritzung vertragen würden.

Sollte sich dieses Verfahren bewähren - und unsere bisherigen, wenn auch nicht sehr zahlreichen Erfahrungen ermutigen uns zu dieser Annahme - so könnte die Behandlungsdauer der Uterusgonorrhoe voraussichtlich noch mehr abgekürzt werden.

Für die Beurteilung der Heilerfolge ebenso wie für die Diagnose der Uterusgonorrhoe sind für uns in erster Reihe die

I) Anmerkung während der Korrektur. In der letzten Zeit nehmen wir von einer Dislokation des Uterus ganz Abstand. 
Resultate der mikroskopischen Sekretuntersuchungen maßgebend.

Selbst bei akuten Fällen ron Uterusgonorrhoe, in welchen die Diagnose - wie erwähnt war - auch aus den klinischen Symptomen (wenigstens in den meisten Fällen) gestellt werden kann, halten wir für notwendig, die klinische Untersuchung mit der mikroskopischen zu ergänzen. Schon bei subakuten, noch mehr aber bei chronischen Fällen ist die mikroskopische Sekretuntersuchung unerlässlich, indem das Ergebnis dieser letzteren dem klinischen Bild keinesfalls immer entspricht.

Wir finden eitrige Ausflüsse, in denen wir trotz des fleibigsten Suchens keine Gonokokken nachweisen können, und umgekehrt Gonokokken in weniger eitrigem, manchmal sogar glasigem Sekret. Wir können also auf mikroskopischem Wege auch dort die Gonorrhoe nachweisen, wo wir aus dem klinischen Bild nicht darauf geschlossen hätten.

Viel schwerer ist die Entscheidung, welchen Standpunkt wir nach dem negativen Ausfall der mikroskopischen Untersuchung einnehmen sollen. In chronischen Fällen kann sowoht das klinische Bild, als auch der mikroskopische Befund große Schwankungen aufweisen. Wir müssen dann entscheiden, ob wir es mit einer virulenten, oder einer, vom Standpunkt der Prophylaxe nicht so wichtigen, residualen Gonorrhoe, oder mit einem anderen katarrhalischen Prozeß zu tun haben. In solchen Fällen beweisen $1-2$ negative Befunde nichts. Wenn wir auch nach mehreren Untersuchungen zu keinem Resultate kommen, so versuchen wir die Gonokokken auf provokatorischem Wege aus ihrem Versteck zum Vorschein zu bringen. Für diesen Zweck haben sich die intrauterinen Einspritzungen von Nat. lygosinat ebenfalls sehr brauchbar erwiesen. Es kann vorkommen, daß wir auch hiernach keine Gonokokken finden; manchmal haben wir aber beobachten können, daß der bishin profuse Ausfluß seinen eitrigen Charakter langsam einbüßt, schleimiger wird, abnimmt, eventuell auch ganz aufbört.

Finden sich im Sekrete, selbst nach öfter wiederholten provokatorischen Einspritzungen keine Gonokokken, so können wir annehmen, daß wir es mit einem postgonorrhoeischen Katarrh, oder einem anderen katarrhalischen Prozeß zu tun haben. Ebensowenig konstatieren wir die Heilung aus dem klinischen Bilde allein, sondern auf Grund des mikroskopischen Befundes. Das Sekret des Zervikalkanals wird zweimal wöchentlich untersucht, am Tage vor der Einspritzung. Für "geheilt“ oder vorsichtiger ausgedrückt, für Gonokokkenfrei halten wir nur jene Patientinnen, bei welchen nach Aussetzen der Therapie wenigstens zwei mikroskopische Untersuchungen negativ ausgefallen sind. 
Mehrere Autoren betonen, daß die aus dem Uterussekrete schon verschwundenen Gonokokken nach der Menstruation in demselben wieder auftreten können. Wir können das ebenfalls bestätigen. In vereinzelten Fällen aber verschwanden die Gonokokken definitiv gerade nach der Menstruation.

Jadassohn (3) hält die Gonorrhoe dann für geheilt, wenn Gonokokken 8-10 Tage nach Aussetzen der lokalen Behandlung trotz sorgfältiger mikroskopischer Untersuchung nicht mehr nachweisbar sind.

Er bemerkt selbst, daß diese 10tägige Pause den Prostituierten durchaus nicht angenehm und schwer durchzuführen ist.

Unsere Patientinnen blieben nach Aussetzen der Behandlung ebenfalls durchschnittlich 8-10 Tage in der Klinik, manchmal auch länger, falls eine anderweitige Erkrankung die Entlassung unmöglich machte. Nur bei denjenigen Patientinnen waren wir durch äufere Umstände genötigt diesen Termin abzukürzen, welche bereits 3 Monate oder über 3 Monate im Hospital lagen. Die regelmäßigen Gonokokkenuntersuchungen wurden einmal wöchentlich auch während dieser Zeit forgesetzt.

Bevor wir an die Schilderung unserer therapeutischen Erfolge schreiten, müssen wir kurz über jene bakteriologischen Versuche und Untersuchungen referieren, welche wir zur Erprobung der gonokokkentötenden und entwicklungshemmenden Wirkung des Natrium lygosinat angestellt haben.

Nach dem Neisserschen, heute schon allgemein angenommenen Prinzip mul3 ein wirklich gutes antigonorrhoisches Mittel bei möglicbster Schonnng der Gewebe eine starke gonokokkentötende Wirkung aufweisen.

In dieser Beziehung nehmen die Silbersalze zweifelsohne den ersten Platz ein, und es versprach interessant zu werden die Wirkung des Natrium lygosinat mit derjenigen des Argentamins zu vergleichen, da dieses letztere bekanntlich sehr hohe - und genau studierte - gonokoklsentötende Wirkung besitzt, und zu intrauterinen Einspritzungen bisher fast auss chile 5 ilich gebraucht wurde.

Vor Beginn der balteriologischen Versuche suchten wir nach einem schnellen und sicheren Kulturverfahren. Bei der Wahl eines für die Reinzüchtung der Gonokokken geeignetsten Nährbodens verursacht bekanntlich nicht so sehr die Metbode, als die Beschaffung der hiezu notwendigen Materialien Schwierigkeiten. In der langen Versuchsreihe, die nach $B u m m s$ gelungenen und auch durch uretbrale Impfungen kontrollierten Gonokokkenzüchtungen ihren Anfang nahm, suchte man nach solchen Näbrböden, anf denen der Gonococcus rasch wächst, und deren Bestandteile leicht zu beschaffen sind, und kam allmählich zur Überzeugung, daß tierische Flüssigkeiten für Nährboden viel weniger geeignet sind, als eiweißhaltige menschliche Flüssigkeiten, wie Blutserum, Hydroceleflüssigkeit, Ascites u. s. w. Uns standen Ascitesflüssigkeit und menschliches Blutserum zur Verfügung. 
Wir stellten unsere Nährböden nach dem von Ki efer (5) angegebenen Verfahren her. $(3.5 \%$ Agar-Agar, $5 \%$ Pepton, $2 \%$ Glyzerin, $0.5 \%$ Na. Cl. mit der gleichen Menge Ascites oder Blutserum gemengt.) Bereits 12 bis 18 Stunden nach der Überimpfung auf diesen Nährboden wuchsen auf demselben deutlich unterscheidbare Kulturen. Da uns viel daran lag, möglichst hochvirulente Kulturen zu erhalten, nahmen wir das zu überimpfende Material nur von an ganz akuter und noch nicht behandelter Gonorrhoe leidenden Patienten, in den meisten Fällen von Männern mit Harnröhrentripper. Obwohl Ascitesglyzerinagar und auch Blutserumglyzerinagar sich als sehr gute Nährböden bewährten, beobachteten wir doch, daß auf den mit Blutserum angerichteten Nährböden dio Kulturen rascher und reichlicher gediehen. Je häufiger die Ascitesflüssigkeit sterilisert wurde, um so schwächere Kulturen entwickelten sich darauf. Die diskontinuierlich sterilisierte Ascitesflüssigkeit zeigt ein blasses, opalisierendes Aussehen. Um das za vermeiden, versuchten wir den Pasteur-Chamberlandschen Filter anzuwenden; wir fanden aber, daß auf Nährböden, welche auf dieser Weise mit filtrierter Ascitesflüssigkeit angefertigt wurden, die Gonokokken gar nicht wuichsen.

Zur Feststellung der Identität der Gonokokken ist der Impfversuch nicht unbedingt notwendig, da schon die Tatsache, daß die Gonokokken auf gewöhnlichen Nährböden nicht gedeihen, hierzu verwertet werden kann. Werden die gezüchteten Mikroorganismen auf gewöhnlichen Nährböden (Agar, Bouillon) überimpft, und gedeihen sie auf denselben, so kann man sicher sein, keine Gonokokken gezüchtet zu haben. Auch haben wir das Gramsche Färbeverfahren verwertet; außerdem besitzten schon die Kulturen ein charakteristisches Aussehen.

Nach Wertheim (14) können sie am leichtesten mit Kulturen von Streptococcus pyogenes verwechselt werden, die ebenfalls eine feine Struktur zeigen. Wenn wir indessen die zweifelhafte Kultur auf flüssigen Nährboden überimpfen, so können wir schon nach wenigen Stunden schöne Kettenformen von Streptokokken beobachten. Auch der Befund von sich schlecht färbenden, degenerierten Formen in Färbepräparaten, welche von 48 stündigen Kulturen stammen, spricht für die Anwesenheit von Gonokokken.

Wir wollten durch unsere Versuche feststellen, in welcher Konzentration und in welcher Zeit das Natr. lygosinat hochvirulente Gonokokken tötet. An denselben Kulturen versuchten wir auch das Argentamin, dessen gonokokkentötende Wirkung schon lange bestimmt ist. Wir unternahmen mit beiden Mitteln parallele Versuche, um ihre Wirkung miteinander vergleichen zu können.

Unsere Versuche machten wir im großen und ganzen nach der Vorschrift von Schäffer und Steinschneider (10). Wir mischten destilliertes Wasser oder Bouillon mit der gleichen Menge Blutserum und überimpften auf diese Mischung die 2 Tage alten Serumagarkulturen. Nach der Überimpfung schüttelten wir die Mischung gut durch, damit die Verteilung eine gleichmäßigere sei, und gaben zu bestimmten Mengen 
dieser Flüssigkeit ebenfalls bestimmte Mengen der Lösung des zu untersuchenden Mittels. Die Konzentration der Lösung wechselten wir je nach den Versuchen.

Als Stammlösung gebrauchten wir eine Lösung von 1: 1000. Hievon gaben wir $1 \mathrm{~cm}^{3} \mathrm{zu} 1 \mathrm{~cm}^{3}$ der erwähnten Gonokokkenmischung und erhielten auf dieser Weise eine Verdünnung von 1:2000. Von dieser Flüssigkeit impften wir dann nach 1, 2, 5 und 10 Minuteu nach vorheriger genauer Durchmischung derselben. Die Resultate der einzelnen Versuche - soweit dieselben mit denselben Kulturen angestellt waren wiesen nebeneinander keine wesentlichen Differenzen auf. Wenn die unten angeführten Daten sich weh nicht ganz mit den Resultaten anderer Autoren decken, so erklärt sich dies aus der überaus wechselnden Widerstandsfähigkeit der Gonokokkenkulturen.

Unsere Resultate waren die folgenden:

\begin{tabular}{|c|c|c|c|c|c|}
\hline & \multirow{2}{*}{$\begin{array}{c}\text { Ver- } \\
\text { dünnung }\end{array}$} & \multicolumn{4}{|c|}{$16-20$ Stunden nach der Impfung } \\
\hline & & 1. & $2^{\prime}$ & $5^{\prime}$ & $10^{\prime}$ \\
\hline \multirow{2}{*}{ Argentamin } & $1: 3000$ & $\begin{array}{l}\mid \text { kleine dir bit } \\
\text { angeorducte } \\
\text { Kolonien }\end{array}$ & $\begin{array}{l}\text { weniger, } \\
\text { noch gomug| } \\
\text { winht }\end{array}$ & 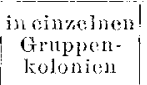 & 0 \\
\hline & $1: 2000$ & $\begin{array}{l}\text { Wenige } \\
\text { Kolonien }\end{array}$ & 0 & 0 & 0 \\
\hline \multirow{3}{*}{ Nat. Gyosinat } & $1: 3000$ & $\begin{array}{l}\text { zum Teil } \\
\text { zusanmon- } \\
\text { fliebonde } \\
\text { Jolonic: }\end{array}$ & weniger & $\begin{array}{l}\text { wenige } \\
\text { Kolonien }\end{array}$ & einige Kol. \\
\hline & $1: 2000$ & $\begin{array}{c}\text { riemlich } \\
\text { dichte } \\
\text { Kolonien }\end{array}$ & weniger & $\begin{array}{l}\text { wenige } \\
\text { Kolonien }\end{array}$ & 0 \\
\hline & i: $1: 1500$ & wenig & 0 & 0 & 0 \\
\hline
\end{tabular}

Wie ersichtlich vernichtete Argentamin in einer Verdünnung von $1: 2000$ schon nach 2 Min. die Kulturen; Nat. lygos in a t setzte bei derselben Verdünnung die Lebensfähigkeit der Kultureu stark herab und tötete dieselben nach $10 \mathrm{Min}$. Die gonokoklkentötende Wirkung des Natr. lygosinat bleibt also etwas hinter derjeuigen des Argentamins zurück, und entspricht - wie aus der Tabelle exsichtlich - eine 1: 1500 Natr. lygosinatlösung einer 1:2000 Argentaminlösung. Daß die klinischen Resultate, welche wir mit Natr. lygosinat erzielten, und welche wir weiter unten schildern, trotzdem keinesfalls hinter dem Argentamin zurückgeblieben sind, erklärt sich daraus, dals das Natr. lygosinat viel weniger als Argentamin reizt, und in fünfmal stärkeren Konzentrationen zu intrauterinen Injelstionen gebraucht werden kann. (Natr. Iygosinat in $5 \%$, Argentamin in $1 \%$ [ $[10 \%$ der käuflichen $]$ Lösung.)

Und nun wollen wir unsere klinischen Resultate kurz beschreiben. 
Von den Krankengeschichten der auf unsere Klinik in dem Zeitraum vom Herbst 1897 bis inkl. 1900 aufgenommenen, an Uterusgonorrhoe leidenden Frauen haben wir 244 verarbeitet. Es wurden so viele Kranke systematisch intrauterin behandelt. Außer diesen lagen noch mehrere, an Uterusgonorrhoe leidende Frauen auf der Klinik, doch wurden dieselben entweder nicht intrauterin behandelt, oder mußte die Behandlung aus irgend einem Grunde ausgesetzt werden. Es mußten einige wegen anderweitigen Erkrankungen, z. B. Tbc. pulmonum, auf die interne Klinik, andere wegen schwerer Adnexerkrankungen auf die gynäkologische Klinik verlegt werden. Ein großer Teil der 244 Kranken lag 2, 3, sogar 4 mal innerhalb der genannten Zeit auf der Klinik. Die intrauterine Behandlung wurde (mit wenigen zu Versuchszwecken gemachten Ausnahmen, wobei Protargol [5-10\% Lösung] Argentum colloidale Credé, Jodtinktur angewendet wurden) im Jahre 1898 mit Argentaminlösung (1 : 10) 1898 sporadisch, 1899 und 1900 systematisch mit 5\% Nat.-lygosinatlösung vorgenommen. (In einem besonders hartnäckigea Falle haben wir mit $1 \%$ Natr.-lygosinatlösung intrauterine Irrigationen vorgenommen.)

Die Argentaminlösung 1:10 wurde bei 119 an Uterusgonorrhoe leidenden Frauen angewendet; davon wurden ausschließlich mit Argentamin behandelt 105, unter diesen blieben 4 ungeheilt. Die übrigen 14 wurden außer Argentamin auch mit anderen Mitteln behandelt. Bei mehreren Kranken, welche trotz andauernder vorheriger Behandlung keine Besserung zeigten, verschwanden die Gonokokken beim Wechsel des Mittels. Unter 8 Kranken, welche früher mit anderen Mitteln vergeblich behandelt waren, verschwanden die Gonokokken nach der Anwendung des Argentamin bei 6; in einem Fall unter diesen war vorher Protargol, bei den 5 anderen Natr. lygosinat angewendet. Bei den 2 anderen Kranken, welche auf Natr. lygos. ungeheilt blieben, hatte auch Argentamin keinen Erfolg. Von 6 Kranken, welche bei Argentaminbehandlung ungeheilt blieben, brachte das Natr. lygos. Heilung in 5 Fällen; die sechste Kranke, bei der Protargol angewendet wurde, blieb ungeheilt. Es wurde demnach bei 12 Kranken von 119 mit Argentamin keine Heilung erzielt.

Die Heilungsdauer der Uterusgonorrhoe bei Argentaminbehandlung zeigt die folgende übersichtliche Zusammenstellung. (Als Heilungstermin setzen wir den Tag, von dem an im Uterussekret keine Gonokokken nachgewiesen werden konnten.) Von den ausschließlich mit Argentamin behandelten Uterusgonorrhoen heilten

innerhalb 20 Tagen 20 Kranke

$\begin{array}{lllll}n & 30 & 25 & 25\end{array}$

zusammen 101 Kranke.

innerhalb 50 Tagen 16 Kranke

Die Heilungsdauer schwankt innerhalb ziemlich weiter Grenzen. In einigen Fällen konnten in dem Sekret schon nach dem 12. Tage keine Gonokokken nachgewiesen werden. Andererseits verschwanden dieselben in einem Falle erst am 81. Tage, in einem anderen gar erst am 115 Tage. Die Mehrzahl der Fälle heilte innerhalb 40 Tagen 
$(663 \%)$. Auch die Zahl der innerhalb 20 Tagen geheilten Fälle ist groß genug (ca. 20\%). In nicht ganz 11\% der Fälle erstreckte sich die Heilungsdauer über 61 Tage.

Die Zahl der intrauterinen Einspritzungen schwankte zwischen 3 und 21. Die Gonokokken verschwanden dauernd

nach 3 Einspritzungen in 3 Fällen, nach 11 Einspritzungen in 4 Fällen

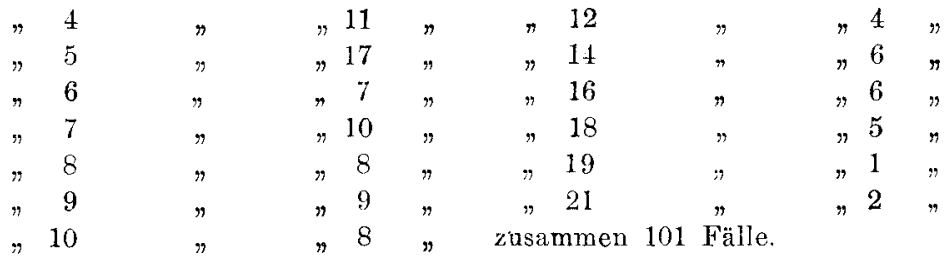

In der Mehrzahl der Fälle (ca 73\%) konnten nash 10 Finspritzungen keine Gonokokken mehr nachgewiesen werden. Mehr als 12 Einspritzungen waren in kaum $20 \%$ der Fälle notwendig.

Die mit dem Natrium lygosinat erzielten Heilerfolge wollen wir in folgendem zusammenfassen. Bis zum Ende des Jahres 1900 wurden mit Natr. lygos. 128 Kranke behandelt; bei 21 dersellen wurden aus später anzuführenden Gründen auch andere Mittel angewendet. Bei den 107 Kranken, die ausschließlich mit Natr. Iygos. bchandelt wurden, blicben 3 ungeheilt, hei den anderen 104 verschwanden dic Gonokokken:

innerhalb 20 Tagen bei 15 Kranken, innerhalb 50 'Tagen bei 15 Kranken

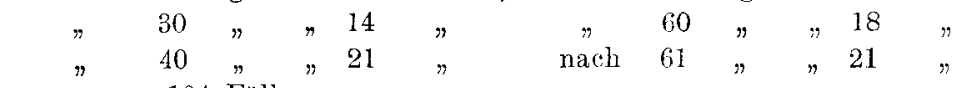

zusammen 104 Fälle.

Innerhalb 40 Tagen sind also bei 50 Patientinnen die Gonokokken verschwunden. Es wird anffallen, dak diese Zahl etwas niedriger ist, als bei den mit Argentamin behandelten Kranken. Der Grund hiefür liegt darin, daß die intrauterine Behandlung gerade in diesen Fällen oft wegen interkurrenten Krankliciten ausgesetzt werden mußte.

So mulite z. B. die intrauterine Behandlung in 3 liallen wegen Gastroenteritis und Ikterus für längere Zeit unterbrochen werden. In einem Falle hinderte ein Absze日, welcher sich in der Genitocruralfalte gebildet hatte, die Einführung des Spiegels; einmal mußte die intrauterine Behandlnng wegen einer Cystitis melrere Wochen lang sistiert werden. Öfters kamen die Patientinuen mit einer Adnexerkrankung und virulenter Uterusgonorrhoe auf die Klinik. In solchen Fällen konnte die intrauterine Bebandlung nur mit äußerster Sorgfalt durchgeführt werden, und falls trotzdem eine Exacerbation - Schmerzen etc. - aufgetreten ist, so mußte die intrauterine Behandlung für längere Zeit ausgesetzt werden. In einem dieser Fälle konnten wir beobachten, daß der ursprünglich männerfaustgroße Adnextumor während der intrauterinen Behandlung bedeutend kleiner geworden jst.

Die anfangs angeführten Fälle sind auch insoferne von Interesse, weil sie beweisen, dali die Bettruhe allein ohne topische Behandlung die 
Uteringonorrhoe - wenigstens in sehr viel Fällen - gar nicht zu beeinflussen vermag. Der gonorrhoische Prozeß zeigte in diesen Fällen nämlich trotz der mehrwöchentlichen, ja manchmal noch länger dauernden Bettruhe - gar keine Veränderung, und die Gonokokken verschwanden erst nach der Wiederaufnahme der intrauterinen Behandlung.

Der größte Teil der ausschließlich mit Natriumlygosinat behandelten Uteringonorrhoen beilte nach 10 intrauterinen Einspritzungen (74 unter 104 - ca. $71 \cdot 2 \%$ ).

Die Gonokokken verschwanden

nach der 2. Einspritzung in 1. Fall, nach der 12. Einspritzung in 3. Fällen

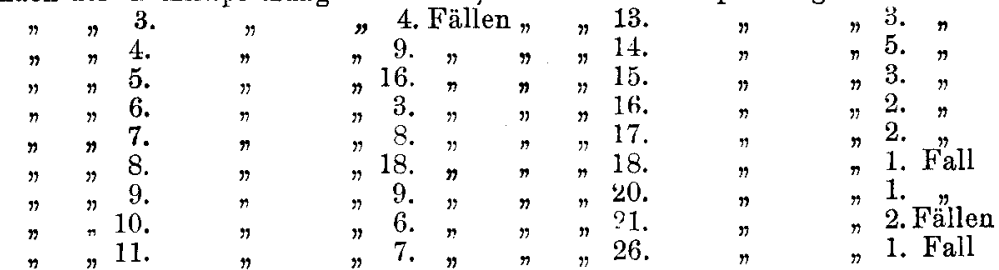

Zusammen $10 \pm$ Fälle.

$\mathrm{Zu}$ diesen 104 Fällen können noch 7 mit Nat. lygos. behandelte und geheilte Fälle gerechnet werden. Von diesen sind 5 vorher mit Argentamin erfolglos behandelt worden, und heilten nach Anwendung von Natrium lygosinat.

2 Kranke hatten 11 , beziehungsweise 8 Einspritzungen mit einer $5 \%$ Lösung von Argent. colloidade erfolglos bekommen und heilten nach 10 beziehungsweise 9 Einspritzungen mit Natr. lygos.

In einzelnen Fällen konnten wir die Gonokokken aus dem Sekret trotz der Behandlung mit Natrium lygosinat nicht verschwinden lassen, ebenso wie wir es früher bei der Besprechung der mit Argentamin behandelten Fälle gesehen haben. Von den ausschließlich mit Natrium lygosinat behandelten Fällen blieben - wie erwähnt - ungeheilt 3. Bei 4 Kranken brachte weder Natr. lygos., noch ein anderes Mittel Heilung. Bei 10 Kranken verschwanden die Gonokokken auf Natr. lygos--Behandlung zwar nicht, wohl aber nach der Anwendung irgend eines anderen Mittels.

Von 128 Kranken, die mit Natr. lygos. behandelt wurden, heilten also 111 , ungebeilt blieben 7 ; es heilten nämlich bei Behandiung mit Natr. lygos. nicht, wohl aber nach Anwendung eines anderen Mittels 10 Kranke.

Der leichteren Übersicht halber fassen wir die Endresultate in folgende Tabelle zusammen:

Das angewendete Mittel: Zahl der Kranken heilten ungebeilt blieben

Natrium lygosinat $\quad 107 \quad 104 \quad 3$

Argentamin

Verschiedene Mittel

(Protargol, Jodtinkt.)

Zuerst Protargol, dann Árgent.

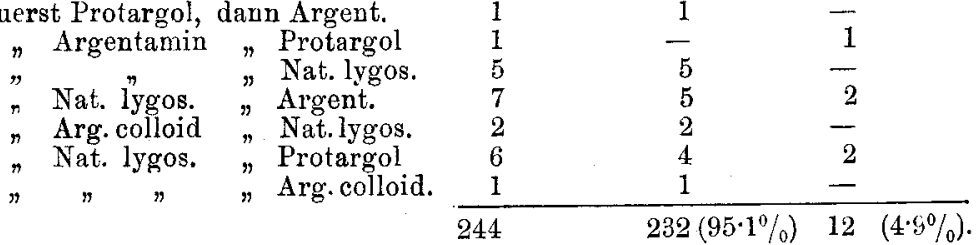


Dieses günstige Resultat ist in der Tat überraschend. Einerseits standen aber unsere Kranke durchschnittlich länger in Behandlung, als die Kranken von Schultz und Lappe (8 u. 14). Andererseits halten auch wir nicht alle Kranke für definitiv geheilt, sind vielmehr überzeugt, dal3 in einem, und vielleicht einem genug ansehnlichen Teil der Fälle die aus dem Sekrete bereits für eine Zeitlang verschwundene Gonokokken in demselben wiederum erschienen wäres, wenn wir die Kranken noch längere Zeit nach dem Aussetzen dor Behandlung hätten beobachten können. Leider steht es nicht in unserer Macht die Prostituierten so lange im Hospital zurückzuhalten, bis eine Rezidive sicher auszuschlieben wäre.

Wie dem aber anch sein mag, so viel steht fest, daß wir durch unsere Behandlung die Uterusgonorrhoe der Prostitaierten in einer sehr ansehnlichen Anzahl der Fälle ganz sicher ausheilen können, wenigstens in dem Sinne, daß die Gonokokken aus dem Sekrete definitiv verschwinden.

Das können wir auch zahlenmäßig beweisen. Wir haltea nämlich, gleich Lappe und Schultz, ebenfalls nur diejenigen Kranken für definitiv geheilt, welche bei der Wiederaufnahme ins Hospital für gonorrhoefrei gefunden wurden.

J. L a p pe hat die Krankengeschichten von 393 Frauen verwertet, welche (in dem Zeitraum von 1892 bis 1896) mit Gonorrhoe behaftet einmal bereits auf der Prostituiertenabteilung des Breslauer Allerheiligen-Hospitals gelegen, wegen Gonorrhoe oder anderweitige Erkrankungen wiederum ins Hospital aufgenommen wurden. Von diesen 393 Kranken fand er bei der zweiten Aufnahme 281 mit Gonorrhoe behaftet. Die anderen 162 waren. gonorrhoefrei. Bei diesen letzteren sind zwischen den beiden Aufnahmen ins Hospital durchschnittlich 5//2 Monate verflossen. Bei diesen konnte also gar nicht angenommen werden, daB ihre Gonorrhoe etwa "im Laufe der Zeit" von selbst ausgeheilt ist. Ebenso wenig würde die Annahme gerechtfertigt sein, dals es sich bei allen der 231 mit Gonorrhoe behafteten Patientinnen um eine Exacerlation der alten Gonorrhoe gehandelt hat. Gine überstandene Gonorrhoe hinterläbt bekanntlich keine Immunität, und besonders die Prostituierten sind sofort nach ibrer Entlassung aus dem Hospital und fortwährend neuen Infektionen ausgesetzt. Davon sielt Lappe jedoch $a b$, und rechnet von diesen 231 fiällen nur 16 zu den 162 ,geheilten ${ }^{4}$. Bei diesen 16 zeigte nämlich bei der Wiederaufnahme die Gonorrhoe eine andere Lokalisation, so daß man sichcr annehmen konnte, daf es sich um eine neue Infektion handelt. Das Minimum der geheilten Fälle beträgt also nach Lappe $162+16=178$, d. l. $45.5 \%$.

I a appe's Daten sind für uns insoferne nicht ohne weiters zu verwerten, indem er den Heilungsprozent der Uterusgonorrhoe nicht separat angegeben lat. Um sehr grobe Differenzen kann es sich aber nicht handeh, da die meisten seiner Patientinnen auch mit Uterusgonorrhoe behaftet waren.

Viel besser können wir Schultz's (13) Daten verwerten, da sich dieselben ebenso, wie die unseren, ansschlieblich auf Uteruscronorrhoen beziehen. Seine behandlungsmethode bestand ebenfalls in intrauterinen Injektionen ( $10 \%$ der käuflicheu Argentaminlösung), welche or in jedem Falle angewendet hatte, obzwar er andererseits bemüht war, die reinen Zervikalgonorrhoen von den Gonorrhoen des Cavum uteri abzusondern (s. oben).

Von 76 Patientinnen, bei welchen Schultz Uterusgonorrhoe festgestellt hat heilten $53=70 \%$. Noch günstiger war der Heilungsprozent bei den rejnen Zervikalgonorrhoen. Unter 81 Kranken blieben nach Schultz nur 5, d. h. $6 \cdot 17 \%$ ungeheilt. Von den geheilt Entlassenen sind 33 ins Hospital wieder aufgenommen worden (Schultz arbeitete auf 
Rónas Abteilung), von diesen fand Schultz $19=57.5 \%$ gonorrhoefrei. Soviel beträgt also das Minimum der Heilung.

Wenn wir nun prozentualisch berechnen, wie viele unserer geheilt entlassener Patientinnen bei der Wiederaufnahme ins Hospital wirklich gonorrhoefrei gefunden wurden, so sehen wir, daß unsere Daten sich mit denen von Schultz ungefähr decken.

Der größte Teil unseres hier bearbeiteten Krankenmaterials besteht aus registrierten, und der übrig gebliebene Teil fast ausschließlich aus clandestinen Prostituierten; also sind es durchwegs solche Frauenspersoven, welche neuen Tripperansteckungen fortwährend ausgesetzt sind.

Unsere hier anzuführenden Daten bedeuten also einen minimalen Prozentsatz der wirklich geheilten Uterusgonorrhoefälle; in der Wirklichkeit ist dieser Prozentsatz gewiß noch größer.

Von den 205 ausschlieBlich mit Argentamin oder Natr. lygos, behandelten und aus der Klinik als "geheilt" entlassenen Kranken wurden 69 wieder aufgenommen. Mehrere davon wurden 3, sogar 4mal in der Klinik behandelt. Die Wiederaufnahme erfolote bei einer großen Anzahl (40 unter 69) binnen 5 Monaten nach der ersten Entlassung, bei einigen sogar in 1 bis 3 Wochen. Von den mit Argentamin behandelten Kranken kamen 31 zurück; bei 15 davon konnten weder bei der Aufnabme, noch im Verlauf der weiteren Behandlung Gonokokken nachgewiesen werden, auch nach provozierender Behandlung nicht. Von den mit Natr. lygos. Behandelten kamen 38 zurück, davon 24 ohne Uterusgonorrhoe. Im ganzen sind also unter 69 wiederaufgenommenen Kranken bei der zweiten Aufnahme 38 gonorrhoefrei gefunden worden, was $55 \%$ entspricht, und zwar entfallen davon auf das Argentamin $48.38 \%$, auf das Natrium lygosinat $63 \cdot 15 \%$.

Vergleichen wir diese Daten mit denen Lappes und Schultzs, so sehen wir, daß dieselben so ziemlich übereinstimmen, indem Lappe als minimalen Prozentsatz der Heilung der weiblichen Gonorrhoe 45.5, $\mathrm{Schultz}$ als denjenigen der Uterusgonorrhoe 57.5 gefunden hat.

Wenn die angeführten Zahlen auch keinesfalls als absolute und frei von jeder Fehlerquelle zu betrachten sind, so glauben wir uns doch berechtigt, auf Grund derselben den Satz aufstellen zu dürfen, $d$ a $\mathfrak{B}$ die Uterusgonorrhoe, wenn sie in der angegebenen Weisebehandelt wird, in einem großen Teil der Fälle definitiv heilt. Wir können dies umso eher behaupten, als wir gefunden haben, daß bei der Mehrzahl der Fälle auch dieklinischen Erscheinungen der Gonorrboe zurückgehen; besonders haben wir das bei den ganz rezenten Fällen beobachten können.

Wir wollen auch jenen Einwänden begegnen, welche unserem therapeutischen Vorgehen entgegengehalten werden könnten. Vor allem müssen wir betonen, daß wir die von Fehling erwähnten schweren Symptome nach dem Gebrauch der Braunschen Spritze bei unseren Patientinnen kein einziges Mal beobachten konnten.

Jene subjektiven Erscheinungen, welche den intrauterinen Injektionen mitunter folgen, sind nicht von großer Bedeutung. Bei jenen Kranken, deren os internum so eng war, daß die Kanüle nur schwer eingeführt werden konnte, traten nach der Einspritzung $1 / 2$ Stunde, manchmal etwas länger dauernde kolikähnliche Schmerzen auf, welche aber bei Bettruhe und Prießnitzumschlägen alsbald vorübergingen. Später, wenn die Einspritzung 
nach vorheriger Erweiterung des os internum vorgenommen wurge, traten solche Krämpfe nicht mehr auf. Das würde dafür sprechen, daß die Spannung des schwer passierbaren os internum den krampfartigen Schmerz auslöste. Das eingespritzte Mittel selbst verursacht selten Schmerzen. Nur nach Argentamininjektionen traten manchmal leichte Schmerzen auf, die sich später steigerten und 6 bis 8, sogar bis 24 stunden dauerten. Bei der Natrium lygosinat-Behandlung traten Schmerzen seltener auf; dieselbe stellen sich dann unmittelbar nach der Injektion ein und erreichen rasch ihren Höhepunkt und gehen meistens obenso rasch besonders auf Bettruhe und Prießnitzsche Umschläge - wieder zurück. Wir haben überbaupt den Eindruck gewonnen, daß die, durch das Natrium lygosinat bedingten subjektiven Erscheinungen lange nicht so vehement waren, wie die nach Argentamin und gingen auch schneller vorüber.

Die subjektiven Erscheinungen waren übrigens nie so ernster Natur, daß wir ihretwegen gezwungen gewesen wären, von der Behandlung ganz abzustehen.

Der zweite, viel gewichtigere Einwand, der gegen die intrauterinen Injektioneu erhoben werden könnte, ist, daß dieselben das Ascendieren der Gonorrhoe befördern, indem einerseits in die eventuell noch sterile Uterushöhle Gonokokken verschleppt werden, andererseits und hauptsächlich aber, indem dadurch gonokokkenhaltiger Eiter in die bishin noch sterilen Tuben gepreßt wird, und Adnexerkrankungen hervorgerufen werden.

Den ersten Teil dieser Frage haben wir bereits beantwortet, indem wir sagten, daß das Os internum unserer Meinung nach nur eine anatomische Grenze zwischen Cervix und Cavum uteri bildet, und da wir vorlänfig keine sichere Methode besitzen, um bei gonokokkenhaltigem Sekret aus dem Cervix das Erkranktsein des Endometriums auszuschließen, so ist es rationeller - wenigstens bei unserem Krankenmaterial - vom Anfang an auch das Cavum uteri zu behandeln. ${ }^{1}$ ) Wir können hier noch hinzufügen, daß es schwer anzunehmen wäre, daß ein stark gonokokkentötendes Mittel in so starker Konzentration, wie wir es gebrauchen, lebensfähige Gonokokken vom Cervix in das Cavum uteri verschleppen könne.

1) Anmerkung während der Korrektur. In der letzten Zeit gehen wir auf der Weise vor, daß wir in solchen Fällen, wo der innere Muttermund schwer durchgängig ist, und keine klinischen oder subjektiven Symptomevorhandensind, welche ein Erkranktsein des Endometriums für wahrscheinlich machten, die erste, eventuell die ersten beiden Injektionen nur in den Cervicalkanal deponieren. In einigen Fällen gelang es uns die Gonokokken aus dem Sekret nach ein- oder zweimaligen Injektion definitiv verschwinden zu lassen. In diesen Fällen hat es sich sicherlich um reine Zervikalgonorrhoen gehandelt. Hierüber werden wir in einer späteren Arbeit referieren. 
Was den zweiten Punkt anlangt, so kann die Möglichkeit nicht von der Hand gewiesen werden, daß durch die intrauterinen Injektionen, wenn sie auch mit starken gonokokkentötenden Mitteln und in starker Konzentration gemacht werden, in vereinzelten Fällen, besonders wenn sie mit der nötigen Sorgfalt und Vorsicht angewendet werden, Adnexerkrankungen hervorgerufen werden. Das können wir uns vielleicht auf die Weise erklären, daß infolge der durch das mechanische Einwirken und Dehnen des Os internum hervorgerufenen Uteruskonzentrationen etwas gonokokkenbaltiger Eiter in das Ostium uterinum tubae gepreßt wird, bevor die intrauterine Injektion stattgefunden hat, bevor also jene Gonokokken mit dem antiseptischen Mittel in Berührung gekommen sind.

Seinerzeit, wenn wir unsere, durch regelmäßige, sachgemäße nnd nicht von uns, sondern einem ganz unparteiischen Gynäkologen angestellte gynäkologische Untersuchungen ergänzte Heilerfolge publizieren werden, werden wir diese Frage näher beleuchten. Soviel können wir aber schon jetzt sagen, daß solche Fälle, wo eine Adnexerkrankung während der intrauterinen Behandlung und scheinbar durch dieselbe entsteht (oder wo eine bereits bestehende Adnexerkrankung durch die intrauterinen Injektionen ungünstig beeinflußt wird) nur die Ausnahme bilden, und das kann kein Grund sein, auf die sonst guten, sogar überraschend gute Wirkungen unserer Therapie zu verzichten. Fs liegt ja im Interesse der Prostituierten selbst, von ihrem Leiden befreit zu werden. Bei wie vielen unter ihnen verhindern wir das Auftreten der Adnexerkrankung dadurch, daß wir ihre Uteringonorrhoe ausbeilen? Sicherlich bei sehr vielen, vielleicht bei den meisten. Ist vielleicht die Gefahr einer Adnexerkrankung nicht bei jeder, an virulenter Uterusgonorrhoe leidender und nicht hospitalisierten Prostituierten - zumal sie sich der Cohabitation auch während der Menstruation nicht enthalten - eine ungemein viel gröBere, als die Gefahr einer Tubeninfektion durch unsere Behandlungsmethode? Es ist doch jedem bekannt. wie häufig Adnexerkrankungen auch bei solchen Prostituierten vorkommen, deren Uterusgonorrhoe noch nie behandelt war.

Das Dogma von der Unheilbarkeit der weiblichen Gonorrhoe, insbesondere der Uteringonorrhoe der Prostituierten, kann nach unserer Meinung nicht mehr aufrechterhalten werden. Dieses Dogma stammt hauptsächlich von den Gynäkologen her, und wir sind uns wohl bewußt, daß unsere Behauptungen bei einer großen Anzahl der Gynäkologen nicht nur keinen Anklang finden, sondern sogar energisch bekämpft werden werden. Der Standpunkt der Venereologen ist aber von dem des Gynäkologen doch etwas verschieden, und zwar liegt diese Verschiedenheit, wie darauf jüngst hauptsächlich N e iss e r (19), später auch Marschalkó (6) hingewiesen haben, entschieden daran, daß auch unser Krankenmaterial ebenfalls ein grundverschiedenes ist.

Das Gonorrhoematerial den Gynäkologen - sagt Marschalkó (l. c.) - bezicht sich zum großen 'Teil auf Ehefrauen, welche in der Ehe mit Tripper angesteckt, zumeist schon eine oder auch mehrere Schwangerschaften durchgemacht haben, und welche meist schon mit, seit längerer Zeit bestehenden, schweren, oder gar mit Adnexerkrankangen komplizierter Uteringonorrhoe zum Arzt gelangen. 
Daß hier also sehr viele, schwer heilbare, ja direkt unheilbare Fälle von Gonorrhoe vorkommen, liegt ja an der Hand. Wir aber haben es zumeist mit ganz jungen, noch keine Graviditäten durchgemachten Personen zu tun, wo also die Krankheit entschieden im recenteren, der Therapie mehr zugänglichen Stadium sich befindet."

Und so bietet die Gonorrhoe der Prostituierten - vorausgesetzt, daß sie rechtzeitig entdeckt und der Behandlung zugeführt wird - vielmehr Aussicht auf Heilung. Mit der Heilung ihrer Gonorrhoe wird nicht nur die Prostituierte von ibrer folgeschweren Erkrankung befreit, sondern auch eine Infektionsquelle beseitigt, also der Prophylaxe der Gonorrhoe ein guter Dienst geleistet.

Bei der Gonorrhoe-Behandlung der Ehefrauen kommt dieser letztere Gesichtspunkt nicht in Betracht.

Außerdem dürfen wir auch das nicht vergessen, da $\beta$, wenn wir auch nicht im stande sind, die Uterusgonorrhoe in jedem Falle zu heilen, durch unsere Behandlung doch wenigstens eine Herabsetzung der Virulenz erzielen können.

Auf Grund des Gesagten glauben wir behaupten zu können, daß es ratsam ist, die Uterusgonorrhoe - - von den sehr akuten, mit vehementen Symptomen einhergehenden Fällen abgesehen - einer lokalen topischen Behandlung zu unterziehen.

Und wenn bei der Behandlung der männlichen Gonorrhoe die Grundsätze Neissers: nicht zuwarten, sondern die Behandlung - mit den nötigen Kautelen - mit antigonorrhoischen Mitteln sofort zu beginnen, sich langsam überall Bahn brechen, so dab das exspektative Vorgehen fast allgemein verlassen wurde und sogar perhorresziert wird, so steht es zu hoffen, daß die Ausichten bezüglich der Therapie der Uterus-Gonorrhoe sich langsam ebenfalls in diese Richtung lenken lassen werdeo.

Wird die Uterushöhle nicht mehr für ein „noli me tangere“ angesehen werden, sondern wenn man sich gegebenen Falls mit der nötigen Vorsicht zu einer intrauterinen Behandlung entschliessen wird, so können wir hoffen, daß das Dogma von der Unheilbarkeit der Uterusgonorrhoe langsam aus der Welt geschaften wird.

Für diese Behandlung haben wir in dem Natrium lygosinat zweifellos ein sehr brauchbares, sogar hervorragendes Mittel. Es leistet innerhalb der Grenzen der Heilbarkeit von der Uterusgonorrhoe ausgezeichnete Dienste.

Es besitzt Eigenschaften, welche ihm auch unter den anerkannten antigonorrhoischen Mitteln einen hervorragenden: Platz sichern. 
Neben seinen hohen antiseptischen Eigenschaften resp. bedeutender gonokokkentötender Wirkung, verursacht es fast gar keine Reizung und schädigt die Gewebe gar nicht. Diese letzte Eigenschaft dürfte geeignet sein, auch die Heilungsdauer der Uterusgonorrhoe $\mathrm{zu}$ verkürzen, indem man versuchen könnte, die intrauterinen Injektionen in kürzeren Intervallen, als es bisher geschehen - vorzunehmen.

Zum Schlusse sei es mir gestattet, meinem verehrten Chef Herrn Prof. v. Marschalkó für Rat und Tat, mit welcher er mir bei der Verfassung dieser Arbeit ununterbrochen zur Seite stand, meinen tiefgefühlten Dank auch an dieser Stelle auszusprechen.

\section{Literatur.}

1. Behrend. Über die Gonorrhoebehandlung Prostituierter. Berliner klin. Wochenschr. 1898. 115.

2. Fehling. Behandlung der Endometritis. Verhandlungen der deutschen Gesellschaft für Gynäkologie. VI. (1895).

3. Jadassohn. Die Gonorrhoe der Prostituierten. Conf. internat. pour la prophylaxie de en syphilis et des maladies vénérinnes. Bruxelles. 1899. Rapp. préliminairs. Deuxième Question 48.

4. Jadass o hn. Über die Behandlung der Gonorrhoe mit Argentum Casein. Arch. f. Dermat. u. Syphilis. XXXII. 179.

5. K i f f r. Bakteriol. Studien zur Frage der weiblichen Gonorrhoe. Beiträge z. Geburtshilfe u. Gynäkologie. Festschr. f. Aug. Martin. 1895. p. 265.

6. v. Marschalko. Ist die Gonorrhoe der Prostituierten heilbar? Berliner klin. Wochenschr. 1902. Nr. 15.

7. v. Marschalkó. Reflexionen über die Prophylaxe der venerischen Erkrankungen. Münchener med. Wochenschr. 1901. Nr. 21.

8. Lappe. Statistische Beiträge zur Gonorrhoe der Prostituierten. Allgem. med. Zentralzeitung. 1897. Nr. 7, 8.

9. Neisser. Über die Gonorrhoebehandlung Prostituierter. Berl. klin. Wochenschr. 1898. 218.

10. Schäffer. Über Desinfektionsversuche an Gonokokken. Verhandlungen $d$. deutschen dermat. Gesellsch. V. Kongreß. 433.

11. Schäffer u. Steins chneider. Über die Widerstandsfähigkeit der Gonokokken gegen Desinfizientien. IV. Kongr. d. deutschen dermat. Gesellschatt zu Breslau. 1894.

12. Schäffer. Über die Bedeutung der Silbersalze für die Therapie der Gonorrhoe. Münchener med. Wochenschr. 1895. Nr. 28, 29.

13. S choltz. Beiträge zur Biologie des Gonococcus (Kultur, Tier experimente etc.). Archiv f. Dermatol. u. Syphilis. XLIX. 3.

14. Schultz, H. Beiträge zur Path. u. Therap. d, weibl. Gonorrhoe. Archiv f. Dermat. Bd. XXXVI. Orvosi Hetilap. 1899. 3. sz.

15. Steinschneider und Schäffer. Zur Biologie des Gonococcus. Berliner klinische Wochenschrift. 1895. Nr. 45.

16. Wertheim. Rezidive und Übertragbarkeit der Gonorrhoe. Wiener klin. Wochenschr. 1894. 24.

17. Wertheim. Die ascendierende Gonorrhoe beim Weibe. Arch. f. Gynäkologie. XLII. 1 .

18. Wertheim. Über Uterusgonorrhoe. Verhandl. der deutschen Ges. f. Gynäkologie. VI. Kongr. 1895. 217.

19. Ne isser. Verbandlungen der 1. Brüsseler internat. Konferenz. Compte rendu des séances. pag. $167 \mathrm{ff}$. 